\title{
Implementação de uma FFT com complexidade multiplicativa abaixo do limitante de Heideman-Burrus
}

\author{
P.A.L. Sá de Melo, H.M. de Oliveira
}

\begin{abstract}
The computational implementation of a new fast algorithm for computing DFT based on a matrix Laurent series [1] is presented via Simulink ${ }^{\mathrm{TM}}$. The arithmetic complexity, expressed by the number of nontrivial real floating-point multiplications, achieves values below the standard HeidemanBurrus bound [2]. The case $N=16$ is presented in details, requiring merely 12 real multiplications and 101 additions.
\end{abstract}

Resumo- A implementação computacional em plataforma Simulink $^{\circledR}$ de uma nova transformada rápida de Fourier com base em Séries de Laurent matriciais [1] é apresentada. A complexidade aritmética, expressa em multiplicações reais nãotriviais, atinge valores inferiores àqueles estabelecidos na cota padrão de Heideman-Burrus [2]. O exemplo $N=16$ é apresentado detalhadamente, com apenas 12 multiplicações reais e 101 adições.

Palavras Chave - algoritmos rápidos, Fourier, FFT, séries de Laurent, cota de Heideman-Burrus.

\section{INTRODUÇÃO}

A transformada de Fourier, contínua ou discreta, desempenha um papel muito importante em diversas áreas da Engenharia, principalmente em processamento de sinais.

Este artigo apresenta uma implementação em Simulink ${ }^{\circledR}$, ferramenta de programação visual do software MATLAB ${ }^{\circledR}$, de um novo algoritmo rápido para o cálculo da Transformada Discreta de Fourier (DFT) [1]. Seja $N$ o número de amostras no domínio do tempo de um sinal $v=\left(v_{n}\right), n=0,1,2, \ldots, N-1$. A DFT do sinal $v$ é dada pela sequência $V=\left(V_{k}\right)$, de comprimento $N$, no domínio da frequência, expressa por:

$$
V_{k}:=\sum_{n=0}^{N-1} v_{n} \exp \left(-\frac{j 2 \pi k n}{N}\right) .
$$

Esta transformada pode ser calculada através de algoritmos rápidos, tais como o algoritmo Cooley-Tukey, e o algoritmo Winograd-Fourier [3].

Em 1986, Heideman investigou a complexidade aritmética da DFT e deduziu cotas inferiores sobre o número de multiplicações complexas necessárias para calculá-la [2]. Para potências de 2, esta cota é simplificada para :

$$
\mu_{D F T}(N)=4 N-2 \log _{2}^{2} N-2 \log _{2} N-4 .
$$

\section{A DFT COMO UMA SÉRIE DE LAURENT MATRICIAL}

de Oliveira, Campello de Souza e de Oliveira propõem, em um artigo submetido neste mesmo Simpósio [1], um

H.M de Oliveira e P.A.L. Sá de Melo são membros do CODEC, Universidade Federal de Pernambuco (UFPE), C.P. 7800, CEP: 50711970, Recife-PE, Brasil. (e-mails: hmo@ufpe.br, pmelo@hotmail.com). algoritmo para cálculo da DFT com base na decomposição da matriz da DFT em série matricial de Laurent [4].

Define-se inicialmente uma matriz $M:=(k . n(\bmod N))$. Define-se também um operador $\chi_{1}$ sobre uma matriz $N \times N$ para $l=0,1,2, \ldots, N-1$, que resulta numa matriz cujos elementos são $\left(\delta_{l, m_{k, n}}\right)$, em que $\delta$ denota o símbolo de Kronecker. Desta forma, são construídas classes $C_{m}$ e as respectivas matrizes $M_{m}$ expressas por:

$$
\begin{gathered}
C_{m}:=\left\{x \in Z_{N} \mid 4 x \equiv 4 m(\bmod N)\right\} ; \\
M_{m}=1 \cdot \chi_{m}(M)-j \cdot \chi_{m+N / 4}(M)-1 \cdot \chi_{m+N / 2}(M)+j \cdot \chi_{m+3 N / 4}(M), \\
m=-\left\lfloor\left(\frac{N}{4}-1\right) / 2\right\rfloor, \ldots,-2,-1,0,+1,+2, \ldots,+\left\lceil\left(\frac{N}{4}-1\right) / 2\right\rceil
\end{gathered}
$$

A matriz da transformada de Fourier pode assim ser avaliada como [1]:

$$
\begin{aligned}
\Re e D F T & =\left\{\Re e\left(M_{0}\right)+\sum_{m=1}^{(N / 4-1) / 2} \Re e\left(M_{m}+M_{-m}\right) \cdot \cos \frac{2 \pi m}{N}\right\} \\
& +\left\{\sum_{k=1}^{(N / 4-1) / 2} \mathfrak{I} m\left(M_{m}-M_{-m}\right) \cdot \sin \frac{2 \pi m}{N}\right\}, \\
\mathfrak{I} m D F T & =\left\{\mathfrak{I} m\left(M_{0}\right)+\sum_{m=1}^{(N / 4-1) / 2} \mathfrak{I} m\left(M_{m}+M_{-m}\right) \cdot \cos \frac{2 \pi m}{N}\right\} \\
& -\left\{\sum_{k=1}^{(N / 4-1) / 2} \mathfrak{R} e\left(M_{m}-M_{-m}\right) \cdot \sin \frac{2 \pi m}{N}\right\} .
\end{aligned}
$$

\section{IMPLEMENTAÇÃO EM SIMULINK ${ }^{\circledR}$ PARA $N=16$}

O algoritmo apresentado foi implementado na plataforma Simulink $^{\circledR}$ para $N=16$. Seguindo os passos do algoritmo, constroem-se as seguintes classes:

$$
\begin{array}{ll}
C_{0}=\{0,4,8,12\} & C_{1}=\{1,5,9,13\} \\
C_{-1}=\{15,3,7,11\} & C_{2}=\{2,6,10,14\} .
\end{array}
$$

A partir destas classes, obtêm-se as seguintes matrizes escalonadas, em que rref indica a forma escalonada padrão, $0_{n}$ indica um bloco de $n$ zeros, e ";" indica uma separação de linhas:

$$
\begin{aligned}
& \operatorname{rref}\left[\mathfrak{R e}\left(M_{0}\right)\right]= \\
& \left\{1,0_{15} ; 0,1,0,1,0,1,0,1,0,1,0,1,0,1,0,1 ;\right. \\
& \left.\quad 0_{2}, 1,0_{3}, 1,0_{3}, 1,0_{3}, 1,0 ; 0_{4}, 1,0_{7}, 1,0_{3}\right\} \\
& \operatorname{rref}\left[\mathfrak{R e}\left(M_{1}+M_{-1}\right)\right]= \\
& \left\{0,1,0_{5},-1,0,-1,0_{5}, 1 ; 0_{3}, 1,0,-1,0_{5},-1,0,1,0_{2}\right\} \\
& \operatorname{rref}\left[\Re e\left(M_{2}\right)\right]= \\
& \left\{0,1,0_{3},-1,0_{3}, 1,0_{3},-1,0_{2} ; 0_{2}, 1,0_{7},-1,0_{5} ;\right. \\
& \left.0_{3}, 1,0_{3},-1,0_{3}, 1,0_{3},-1 ; 0_{6}, 1,0_{7},-1,0\right\} \\
& \operatorname{rref}\left[\mathfrak{I m}\left(M_{1}-M_{-1}\right)\right]= \\
& \left\{0,1,0_{5},-1,0,-1,0_{5}, 1 ; 0_{3}, 1,0,-1,0_{5},-1,0,1,0_{2}\right\} \\
& \operatorname{rref}\left[\mathfrak{I m}\left(M_{2}\right)=\right. \\
& \left\{0,1,0_{3},-1,0_{3}, 1,0_{3},-1,0_{2} ; 0_{2}, 1,0_{7},-1,0_{5}\right\} .
\end{aligned}
$$


As componentes do vetor de entrada são combinadas como indicado pelas linhas das matrizes escalonadas e ponderadas de acordo com a equação 3 (c.f. Figura 1.a). Deve-se então encontrar, para cada linha das matrizes da série, quais combinações lineares das linhas das matrizes escalonadas resultam nessas linhas. Estas combinações estão expostas na Figura 1.b, para o cálculo da parte real da DFT.

Tabela 1. Complexidade aditiva do algoritmo FFT baseado em série matriciais de Laurent para $N=16$.

\section{Número de adições reais ponto-flutuante}

\begin{tabular}{l|c} 
Combinações com base nas matrizes escalonadas & 42 \\
Avaliação das componentes reais & 39 \\
Avaliação das componentes imaginárias & 20 \\
\hline Total & 101
\end{tabular}

A complexidade aditiva para o algoritmo apresentado é $\alpha(16)=101$, podendo ainda ser reduzida através do aproveitamento de simetrias adicionais que surgem. A complexidade multiplicativa é $\mu(16)=12$, valor abaixo do limitante de Heideman-Burrus para comprimento 16, que é 20 (equação 2). A complexidade obtida é inferior à calculada pela cota em oito multiplicações. Isto se justifica devido à presença de componentes do espectro nas freqüências de $\pm \pi / 4 \mathrm{e} \pm 3 \pi / 4$, cujas funções seno e cosseno são idênticas, reduzindo o número de multiplicações em quatro unidades, e também devido ao surgimento de linhas idênticas nas matrizes escalonadas no cálculo da parte real e parte imaginária da transformada. A extensão do procedimento para outros comprimentos pode ser vista no artigo companheiro [1].

\section{CONCLUSÕES}

Um novo algoritmo rápido (FFT) para calcular a Transformada Discreta de Fourier (DFT) foi implementado em Simulink ${ }^{\circledR}$, descrevendo as etapas e corroborando que é possível atingir um número de multiplicações pontoflutuante menor do que aquele preconizado pela cota inferior de Heideman. O procedimento é sistemático e fácil de reproduzir, permitindo manipular com maior variedade de comprimentos de bloco do que o tradicional radix-2 [3]. Comenta-se sobre argumentos explorados na diminuição da complexidade, os quais são válidos para outros comprimentos de blocos. Mas o objetivo específico é validar o algoritmo [1] proposto, mostrando que são possíveis implementações de complexidade abaixo do limitante. As simetrias existentes indicam que há muito a se fazer na concepção de hardware dedicados. Esta nova FFT é de fácil implementação, seja em DSP ou circuitos integrados, sendo atrativo explorar implementações em FPGA, dado o paralelo entre Simulink e FPGA.

\section{AGRADECIMENTOS}

Este trabalho recebeu apoio financeiro parcial do Conselho Nacional de Desenvolvimento Científico e Tecnológico (CNPq).

\section{REFERÊNCIAS}

[1] de Oliveira, H.M., Campello de Souza, R.M., and de Oliveira R.C., A Matrix Laurent Series-based Fast Fourier Transform for Blocklengths $\mathrm{N} \equiv 4(\bmod 8)$, XXVII Simpósio Brasileiro de Telecomunicações SBrT, Blumenau, SC, 2009 companion paper (submitted).

[2] Heideman, M.T., Burrus, C.S., On the Number of Multiplications Necessary to Compute a length- $2^{\mathrm{n}}$ DFT, IEEE Trans. Acoust., Speech, Signal Processing, vol.34,pp.91-95, 1986.

[3] Blahut, R. E., Fast Algorithms for Digital Signal Processing, Addison-Wesley, 1985.

[4] Adel-Ghaffar, K.A.S., Long Division from Laurent Series Matrices and the Optimal Assignment Problem, Linear Algebra and its Application, vol.280, No.2-3, Sept pp.189-197, 1998

\section{APÊNDICE}

O código fonte para a implementação simulink encontra-se disponível (freeware) na URL: http://www2.ee.ufpe.br/codec/Procedure_FFT.htm
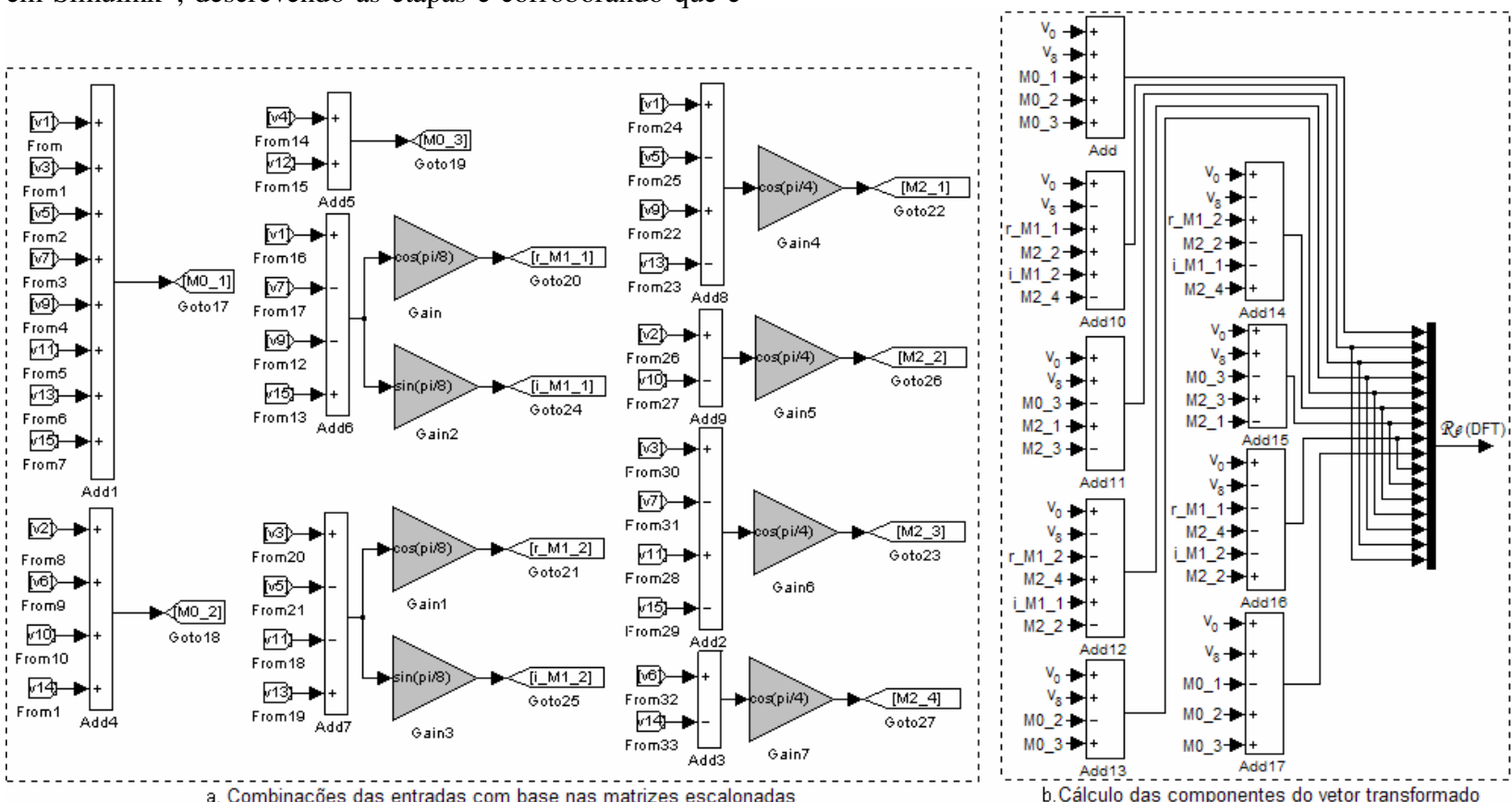

a. Combinaçôes das entradas com base nas matrizes escalonadas

Figura 1. Implementação em Simulink do cálculo da parte real da DFT ( $N=16)$ com uso do algoritmo FFT apresentado. Os blocos triangulares (Fig.1.b) destacam as 8 multiplicações reais, que, somadas com outras 4 do cálculo da parte imaginária, resulta em um total de 12 multiplicações reais pontoflutuante, número inferior às 20 multiplicações da cota de Heideman-Burrus para $N=16$. 